\title{
Síntese e caracterização de novos complexos com a L-butionina sulfoximina
}

\author{
Pedro G. Esquezaro*, Carlos M. Manzano, Pedro P. Corbi.
}

\section{Resumo}

Complexos de $\mathrm{Pd}(\mathrm{II})$ e $\mathrm{Pt}(\mathrm{II})$ contendo os ligantes mistos L-butionina sulfoximina e fenantrolina foram sintetizados e caracterizados por análises químicas e espectroscópicas. Os resultados das análises elementar e termogravimétrica permitiram propor as composições $\left[\mathrm{Pd}\left(\mathrm{C}_{20} \mathrm{H}_{25} \mathrm{~N}_{4} \mathrm{O}_{3} \mathrm{~S}\right)\right] \mathrm{PF} 6$ e $\left[\mathrm{Pt}\left(\mathrm{C}_{20} \mathrm{H}_{25} \mathrm{~N}_{4} \mathrm{O}_{3} \mathrm{~S}\right)\right] \mathrm{PF} 6$ para os complexos. Por espectroscopia na região do infravermelho foi possível sugerir a coordenação do aminoácido ao metal pelo grupo carboxilato e pelo grupo $\mathrm{NH}_{2}$. Para o complexo de $\mathrm{Pd}(\mathrm{II})$, foram realizadas análises por ressonância magnética nuclear e por espectrometria de massas, que reforçam a composição proposta, bem como os sítios de coordenação dos ligantes ao metal.

\section{Palavras-chave:}

Complexos metálicos, L-butionina sulfoximina, antitumoral

\section{Introdução}

Complexos metálicos são utilizados tanto no diagnóstico quanto no tratamento das mais diversas doenças. O maior exemplo é o uso da cisplatina, que é um complexo de $\mathrm{Pt}(\mathrm{II})$, como agente antitumoral. No entanto, sua aplicação é restrita devido a diversos fatores, como nefrotoxicidade e neurotoxicidade ${ }^{[1]}$. Como alternativa, novos complexos de $\mathrm{Pt}(\mathrm{II})$ estão sendo pesquisados, assim como os complexos de $\mathrm{Pd}(\mathrm{II})$, metal que apresenta características semelhantes a platina. Entretanto, os complexos de paládio exibem uma maior reatividade quando comparados aos seus análogos de platina ${ }^{[1]}$.

A L-butionina sulfoximina (bso), é um aminoácido sintético capaz de atuar na regulação da concentração de glutationa no organismo[2], além de apresentar alguma atividade frente células de melanoma in vitro ${ }^{[3]}$. O objetivo deste trabalho foi utilizar a L-butionina sulfoximina como ligante para sintetizar novos complexos de $\mathrm{Pd}(\mathrm{II})$ e $\mathrm{Pt}(\mathrm{II})$, com o auxílio de co-ligantes como a o-fenantrolina, e posteriormente avaliar suas atividades sobre células tumorais.

\section{Resultados e Discussão}

Os precursores de $\mathrm{Pd}(\mathrm{II})$ e $\mathrm{Pt}(\mathrm{II})$ foram preparados adicionando a o-fenantrolina às soluções aquosas de $\mathrm{K}_{2} \mathrm{PdCl}_{4}$ ou $\mathrm{K}_{2} \mathrm{PtCl}_{4}$. As soluções permaneceram sob refluxo por $3 \mathrm{~h}$ no caso do precursor de $\mathrm{Pd}$ (II) e $24 \mathrm{~h}$ no caso do precursor de $\mathrm{Pt}(\mathrm{II})$. Posteriormente, os precipitados resultantes foram separados por filtração, lavados com água e acetona e secos sob $\mathrm{P}_{4} \mathrm{O}_{10}$. Em seguida, os complexos com butionina sulfoximina foram sintetizados em meio aquoso conforme Figura 1. Os complexos de coloração amarela foram separados por filtração e secos em dessecador. Para o complexo de $\mathrm{Pd}(\mathrm{II})$ a composição encontrada foi $\left[\mathrm{Pd}\left(\mathrm{C}_{20} \mathrm{H}_{25} \mathrm{~N}_{4} \mathrm{O}_{3} \mathrm{~S}\right)\right] \mathrm{PF}$. Anal. Calc. (\%): C, 36,79; $\mathrm{H}, 3,86$; N, 8,58. Exp. (\%): C, 36,01; H, 3,63; N, 8,08. IV $\left(\mathrm{cm}^{-1}\right)$ : $v \mathrm{C}=\mathrm{O} 1664, v \mathrm{NH}_{2} 3216$ e 3065. Para o complexo de $\mathrm{Pt}(\mathrm{II})$, obteve-se a composição $\left[\mathrm{Pt}\left(\mathrm{C}_{20} \mathrm{H}_{25} \mathrm{~N}_{4} \mathrm{O}_{3} \mathrm{~S}\right)\right] \mathrm{PF}_{6}$, Anal. Calc. (\%): C, 32,39; H, 3,40; N, 7,56. Exp. (\%): C, 31.74; $\mathrm{H}, 3,11 ; \mathrm{N}, 6,86$. IV $\left(\mathrm{cm}^{-1}\right): v \mathrm{C}=\mathrm{O} 1675$ e $v \mathrm{NH}_{2} 3216,3099$. O espectro na região do infravermelho da bso em comparação aos complexos indica a coordenação do ligante pelo carboxilato e pelo amino grupo. No caso do complexo de $\mathrm{Pd}(\mathrm{II})$, as análises por ressonância magnética nuclear (RMN) de ${ }^{13} \mathrm{C}$ e $\left\{{ }^{15} \mathrm{~N},{ }^{1} \mathrm{H}\right\} \mathrm{HMBC}$ (heteronuclear multiple bond correlation) em dmso- $\mathrm{d}_{6}$, confirmaram a coordenação do ligante ao metal pelo oxigênio do grupo carboxilato e pelo nitrogênio do grupo amino. No espectro de massas, observa-se 0 íon molecular $\left[\mathrm{Pd}\left(\mathrm{C}_{20} \mathrm{H}_{25} \mathrm{~N}_{4} \mathrm{O}_{3} \mathrm{~S}\right)\right]^{+}$de $\mathrm{m} / \mathrm{z}=507.0546$ (erro = 28 ppm), o que confirma a composição proposta.

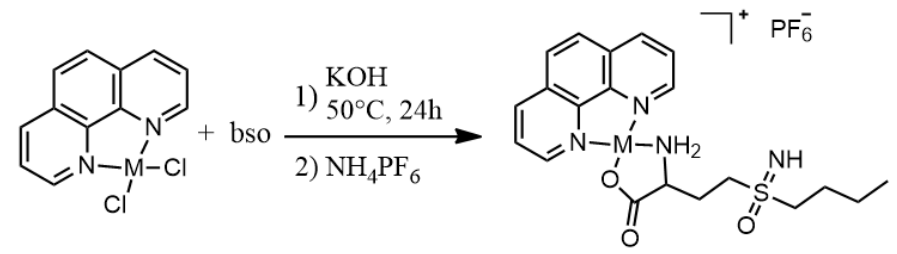

Figura 1. Procedimento de síntese e estrutura proposta dos complexos ( $\mathrm{M}=\mathrm{Pd}$ ou $\mathrm{Pt})$.

A caracterização do complexo de $\mathrm{Pt}(\mathrm{II})$ está em andamento.

\section{Conclusões}

Foram sintetizados dois complexos inéditos de $\mathrm{Pt}(\mathrm{II})$ e $\mathrm{Pd}$ (II) com butionina sulfoximina e o-fenantrolina. As composições dos complexos foram determinadas por análise elementar. O complexo de $\mathrm{Pd}(\mathrm{II})$ foi amplamente caracterizado por espectroscopia no infravermelho, espectrometria de massas e ressonância magnética, sendo possível propor a estrutura apresentada na Figura 1. Estudos das atividades citotóxicas dos complexos sobre células tumorais serão realizados futuramente, comparando os resultados com aqueles descritos na literatura para a cisplatina.

\section{Agradecimentos}

FAPESP (2015/25114-4) e CNPq (PIBIC e Universal 442123/2014-0).

[1] Medici, S.; Peana, M.; Nurchi, V. M.; Lachowicz, J. I; Crisponi, G.; Zoroddu, M. A. Coord. Chem. Rev., 2015, 284, 329-350;

[2] Griffith, O. W. J. Biol. Chem., 1982, 257, 13704-13712.

[3] Prezioso, J. A.; FitzGerald, G. B.; Wick, M. M. J. Invest. Dermatol., 1992, 99, 289-293. 